\title{
AN INTEROPERABLE ARCHITECTURE FOR AIR POLLUTION EARLY WARNING SYSTEM BASED ON SENSOR WEB
}

\author{
F. Samadzadegan ${ }^{\text {a }}$, H. Zahmatkesh ${ }^{\text {a, }}$, M. Saber ${ }^{a}$, H. J. Ghazi khanlou ${ }^{\text {b }}$ \\ ${ }^{a}$ Dept. Of Surveying and Geomatics Eng., Engineering Faculty, University of Tehran, Tehran, Iran \\ (samadz, h.zahmatkesh, m.saber)@ut.ac.ir \\ ${ }^{\mathrm{b}}$ MAS Research \& Development Company, Tehran, Iran \\ Houman.ghazi@masgie.com
}

Commission IV, WG II/8

KEY WORDS: Sensor web, Air Quality, OGC, SWE, WPS, Monitoring System

\begin{abstract}
:
Environmental monitoring systems deal with time-sensitive issues which require quick responses in emergency situations. Handling the sensor observations in near real-time and obtaining valuable information is challenging issues in these systems from a technical and scientific point of view. The ever-increasing population growth in urban areas has caused certain problems in developing countries, which has direct or indirect impact on human life. One of applicable solution for controlling and managing air quality by considering real time and update air quality information gathered by spatially distributed sensors in mega cities, using sensor web technology for developing monitoring and early warning systems. Urban air quality monitoring systems using functionalities of geospatial information system as a platform for analysing, processing, and visualization of data in combination with Sensor Web for supporting decision support systems in disaster management and emergency situations. This system uses Sensor Web Enablement (SWE) framework of the Open Geospatial Consortium (OGC), which offers a standard framework that allows the integration of sensors and sensor data into spatial data infrastructures. SWE framework introduces standards for services to access sensor data and discover events from sensor data streams as well as definition set of standards for the description of sensors and the encoding of measurements. The presented system provides capabilities to collect, transfer, share, process air quality sensor data and disseminate air quality status in real-time. It is possible to overcome interoperability challenges by using standard framework. In a routine scenario, air quality data measured by in-situ sensors are communicated to central station where data is analysed and processed. The extracted air quality status is processed for discovering emergency situations, and if necessary air quality reports are sent to the authorities. This research proposed an architecture to represent how integrate air quality sensor data stream into geospatial data infrastructure to present an interoperable air quality monitoring system for supporting disaster management systems by real time information. Developed system tested on Tehran air pollution sensors for calculating Air Quality Index (AQI) for CO pollutant and subsequently notifying registered users in emergency cases by sending warning E-mails. Air quality monitoring portal used to retrieving and visualize sensor observation through interoperable framework. This system provides capabilities to retrieve SOS observation using WPS in a cascaded service chaining pattern for monitoring trend of timely sensor observation.
\end{abstract}

\section{INTRODUCTION}

Air pollution is one of the most important urban and environmental problems caused by human activities that threaten human health (Alesheikh et al 2005). Development of new sensor, wireless and mobile communication technologies offers effective ways for environmental monitoring and disaster management. One of these new technologies is sensor web, which has remarkable significance for science, environmental monitoring, disaster management, industrial controls and many other domains of activity (Markovic et al 2009). Goal of the sensor web research area is to make heterogeneous sensor resources available through Web applications. Several applications using the benefits of Sensor Web to present coherent infrastructure for sharing, discovering, and accessing sensor data in an interoperable, platform-independent and uniform way (Bröring et al 2011). Considering importance and necessity of control and management of air pollution in megacities like Tehran, it is essential to design an interoperable system for monitoring and alerting air quality status in support of disaster managers to make effective decision in emergency situations. Accessing real time sensor observations is often crucial in emergency situations and disaster management (Simonis et al 2011) which leads to interoperability challenge. Diversity and heterogeneity of sensors and their observations and on the other hand different information providers are the main challenge in accessing real time sensor observation in emergency situations.

This paper using sensor web enablement framework of OGC for implementing an urban air quality monitoring system to acquire interoperability challenge. Proposed system, utilizes event processing methods, provides real-time processing capabilities using Standard GeoProcessing services (WPS), and event filtering on sensor data streams for emergency situation discovery. Designed system SES provides event processing capabilities for discovering emergency status from sensor data stream as an input of event detection process.

\footnotetext{
* Corresponding author.
} 


\section{SENSOR WEB}

Sensor Web consists of a system of intra-communicating, spatially distributed sensor pods that can be deployed to monitor and explore new environments

Sensor network is network of many spatially distributed sensors which are computer accessible and deployed for monitoring conditions at different location. A Sensor web is consists of web accessible and discoverable sensor networks that made sensors and their observation accessible and discoverable through the web using standard protocols and application programming interfaces.

OGC as a responsible organization for spatial standards introduce Sensor Web Enablement framework for implementing sensor web idea by codifying standard web services and data encodings. SWE services are designed to enable discovery of sensors and capabilities, Sharing and retrieval of sensor observations, and also subscription to alerts, and tasking of sensors to control observations. Currently, the SWE framework consists of six standards comprising data models and service interfaces.

These service interfaces consists of SOS, SES, SPS, WNS.

This framework consists of following standard web services:

- $\quad$ SOS is responsible for requesting, filtering, and Retrieving observations and sensor system information.

- $\quad$ SES provide capabilities to monitor sensor Observations for discovering critical situations and publishing and subscribing to alerts from sensors.

- WNS is standard service asynchronous delivery of

Messages or alerts from SAS and SPS web services and other elements of service workflows.

- $\quad$ SPS is a Standard web service interface for requesting

User-driven acquisitions and observations. This is the intermediary between a client and a sensor collection management environment.

Also SWE framework provides following standard encodings:

- O\&M is a Standard models and XML Schema for

Encoding observations and measurements from a sensor, both archived and real-time. Standard

Sensor Model Language (SensorML) is a models and XML Schema for describing sensors systems and processes; provides information needed for discovery of sensors, location of sensor observations, processing of low-level sensor observations, and listing of taskable properties (Botts et al 2006).

\section{RELATED WORKS}

The first time sensor web was introduced in NASA JPL laboratory and used for accessing sensors through web for exploring unknown places (Delin et al 1999).

Markovic et al. developed river water pollution monitoring and alert system using OGC Standard services and a DMA which was responsible for executing automated spatial queries, data acquisition from sensors, and deciding and acting based on the set of operator demands or programming logics (Markovic et al 2009).

Henneböhl, et al proposed an OGC web service architecture for near real-time interpolation of air quality over Europe by utilizing Web Processing Service (WPS) for interpolating sensor observations and using Web Coverage Service (WCS) for storing interpolation process results(Henneböhl et al 2009). Everding et al. presented an architecture for combining geospatial data and real-time sensor data for calculating an Integrated Health Index (IHI) (Everding et al 2010). In this architecture they integrate SWE and geoprocessing services by using two Sensor Event Service (SES) instances and a WPS for determining of the IHI in certain areas. Retch et al. developed a system for spatio-temporal aggregation of European air quality observations using SWE services for storing and retrieving sensor observation and WPS for processing air quality observation (Stasch et al 2011). Finally standardised GeoSensor webs for integrated urban air quality monitoring is presented by Resch et al. for monitoring urban air quality and discovering emergency situation by processing sensor data stream (Resch et al 2012). So many research has been done in designing and developing monitoring systems, but steel the challenge of interoperability and real time monitoring is not solved properly. In this research we design a comprehensive system which all of it's component is used OGC standard and the first step of monitoring which is gathering sensor observation are solved by standardization services.

\section{PROPOSED AIR POLLUTION MONITORING SYSTEM}

This research provides a standard air quality monitoring web based system that utilizing internet as scaffold to establish a distributed component system that helps disaster managers to mitigate the devastating impacts of air pollution. The aim is to use the sensor technologies and standard frameworks for creating an interoperable system for monitoring and alerting air quality information for supporting disaster management systems. As presented in figure 1, developed system uses OGC SWE Standard component to addressing interoperability and flexibility challenges in disaster management systems.

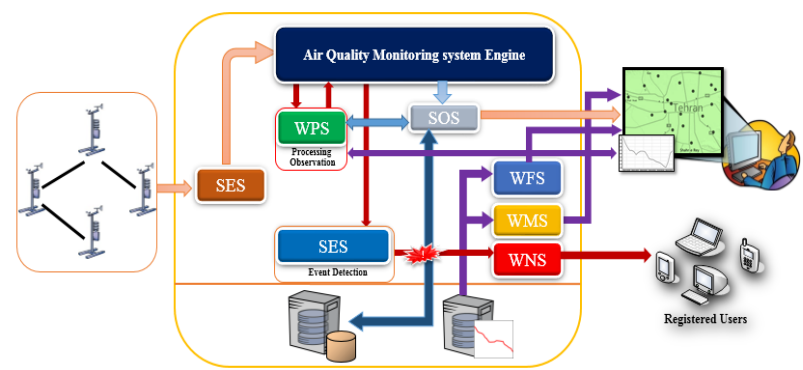

Figure 1. Air Quality monitoring system Standard component and their relation

Air pollution monitoring sensors are responsible for measuring air pollutants such as: $\mathrm{O}_{3}, \mathrm{CO}, \mathrm{PM}, \mathrm{SO}_{2}$ and $\mathrm{NO}_{2}$. In proposed system after measuring the amount of air pollutants, the data is transferred to a central station for further processes. Central station analysing observations and computing air quality index (AQI) which specifies the air quality status for every pollutant. Finally by monitoring AQI extracted from observations, emergency situations are discovered and notified to the authorities.

Developed system using SWE technology for managing heterogeneous sensors in an interoperable way to enable disaster management system using this stream of data as a valuable information to bring update, real time and on-line information resource for making appropriate decisions in case of emergency situations. Developed system calculate AQI from 
measured pollutant. AQI is an index for reporting air quality and its impact on human health based on air pollutants. It is consist of 6 categories, which each category represents by specific colour to indicate level of health concern to authorities and public (Khedo et al 2009). For calculating AQI, air pollutant concentration and AQI values are classified as shown in table1

\begin{tabular}{|c|c|c|c|c|}
\hline O3 & PM10 & CO & SO2 & AQI \\
\hline $0.000-0.064$ & $0-54$ & $0.0-4.4$ & $0.000-0.034$ & $0-50$ \\
$0.065-0.084$ & $55-154$ & $4.5-9.4$ & $0.035-0.144$ & $51-100$ \\
$0.085-0.104$ & $155-254$ & $9.5-12.4$ & $0.145-0.224$ & $101-150$ \\
$0.105-0.12$ & $255-354$ & $12.5-15.4$ & $0.225-0.304$ & $151-200$ \\
$0.125-0.374$ & $355-424$ & $15.5-30.4$ & $0.305-0.604$ & $201-300$ \\
& $425-504$ & $30.5-40.4$ & $0.605-0.804$ & $301-400$ \\
& $505-604$ & $40.5-50.4$ & $0.805-1.004$ & $401-500$ \\
\hline
\end{tabular}

Table 1- Breakpoints of Sensor Observation and for Calculating AQI

AQI is calculated from air pollutant concentration by equation 1. In this equation $B P_{h i}$ and $B P_{l o}$ for each pollutant and also $I_{h i}$ and $I_{l o}$ as AQI range are extracted from Table 1. The concentration is the observation of air pollutant sensors.

$$
A Q I=\frac{I_{h \mathrm{t}}-I_{l o}}{B P_{h \mathrm{r}}-B P_{l o}}\left(\text { Concentration }-B P_{l o}\right)+I_{l o}
$$

Calculated AQI values are classified as shown in table 2. For visualizing air quality status. This is suitable for real time warning in emergency cases.

\begin{tabular}{|c|c|l|}
\hline $\begin{array}{c}\text { Air Quality } \\
\text { Index Levels of } \\
\text { Health Concern }\end{array}$ & $\begin{array}{c}\text { Numerical } \\
\text { Value }\end{array}$ & \multicolumn{1}{|c|}{ Meaning } \\
\hline Good & 0 to 50 & $\begin{array}{l}\text { Air quality is considered } \\
\text { satisfactory, and air pollution } \\
\text { poses little or no risk } \\
\text { Air quality is acceptable } \\
\text { Members of sensitive groups may } \\
\text { experience health effects. The } \\
\text { general public is not likely to be } \\
\text { affected. } \\
\text { Unhealthy } \\
\text { for Sensitive } \\
\text { Groups } \\
\text { Everyone may begin to } \\
\text { experience health effects } \\
\text { Health warnings of emergency } \\
\text { conditions. The entire population } \\
\text { is more likely to be affected } \\
\text { Health alert: everyone may } \\
\text { experience more serious health } \\
\text { effects }\end{array}$ \\
$\begin{array}{c}\text { Very } \\
\text { Unhealthy }\end{array}$ & 101 to 150 to 100 \\
Hazardous & 301 to 500 to 300 \\
\hline
\end{tabular}

Table 2- AQI color and their meanings

Monitoring and event detection workflow of the urban air quality monitoring system which based on standard spatial web services that implemented in a service oriented architecture shown in figure 2 .

Initially, sensor observations are modeled into O\&M structure as a standard encoding using standardization service in order to provide interoperability between heterogeneous sensor data streams. Then system automatically sends information to SES service. This service forwards the extracted information to air quality monitoring engine, which is a middleware that integrates SWE and geoprocessing services. Air quality monitoring engine invokes SOS for storing observations, and then calculates AQI by calling WPS. Calculated AQI sends to SES for monitoring and detect air quality emergency status. Finally, SES using predefined filters detects emergency situation and informs the authorities by utilizing WNS.

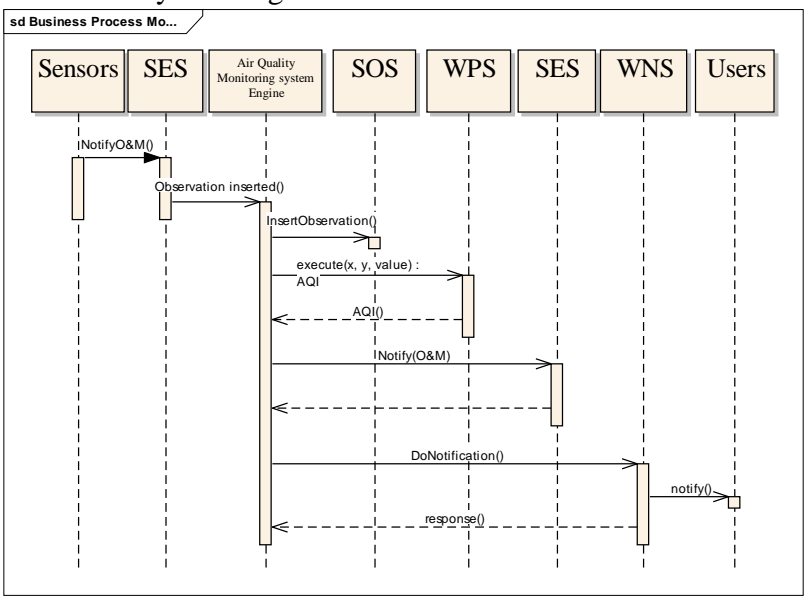

Figure 2. Air pollution monitoring system Sequence

\section{EXPERIMENTS AND RESULTS}

In order to control and manage the air pollution in Tehran, this system has taken benefits of in-situ sensors which are deployed in twenty monitoring stations to cover all pollutant prone areas. For supporting disaster managers to make effective decision in emergency air pollution situation, a GeoPortal (figure 3) for monitoring air pollution developed.

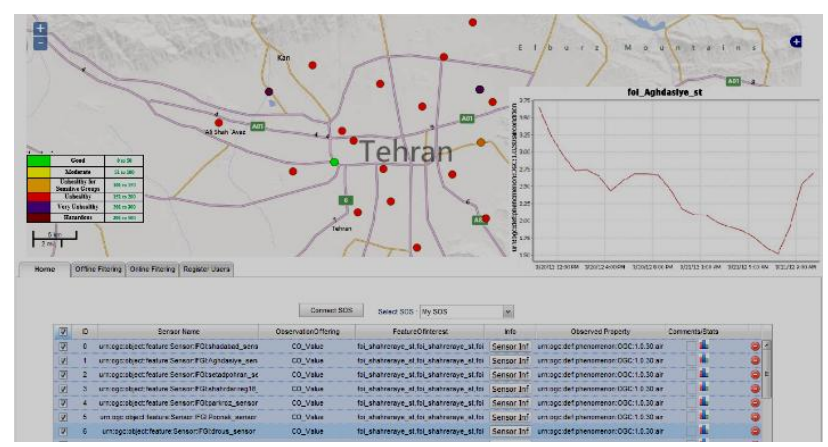

Figure 3. Sensor web geoportal for implementing monitoring air pollution system

In this GeoPortal the observation visualized by standard color for early warning. Figure 3.

This GeoPortal provides capabilities for accessing sensor observation from SOS using various filters as depicted in figure 4 , as well as visualizing them in a time series chart to give managers a better view of air quality status.

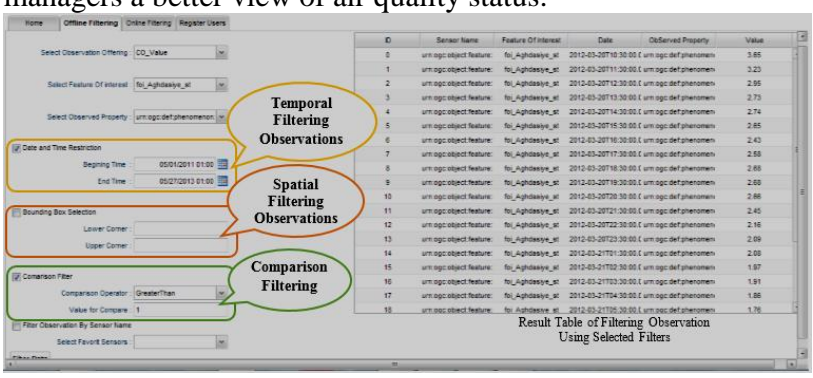


Figure 4. Accessing sensor observation using various filters

For the implementation of described system open source web service software and java technology have been used to analyze the sensor observations from twenty monitoring station in Tehran for discovering and notifying emergency status. This system required standard spatial web services to increase interoperability, flexibility and reusability of system in any other monitoring application. For implementing such system, 52North web service implementation for SOS, WPS, WNS and SES which are the key component of developed system are utilized. In developed system SOS is used for managing Tehran air quality monitoring sensor for storing and retrieving sensor observations ( $\mathrm{Na}$ et al 2007). In this system WPS as a processing unit that provide spatial processes is responsible for calculating AQI (Schut et al 2007).

Besides in this system time series observations of sensors are visualized in charts by createTimelyChart process deployed in WPS service. This service capable of chaining services in cascaded pattern for retrieving observation from SOS and process this observations (Schaeffer et al 2009). As depicted in figure 5, WPS retrieves time series observations from SOS in a cascaded chaining pattern to provide timely chart for following trend of air quality observation from selected sensor in any required time period.

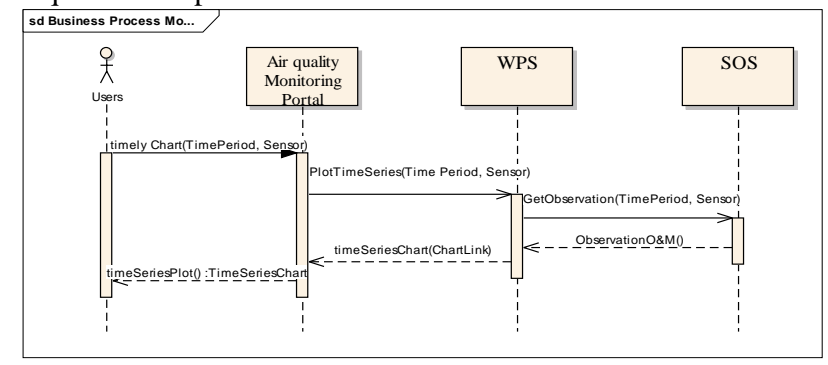

Figure 5-Create Time Series chart From Observation

The key component of this system is SES, which let clients subscribe for observations available at the service. Also performs filtering of sensor data streams based on predefined filtering criteria in subscription request. Filters can be applied on single observations or observation streams, to aggregating observations and extract higher-level information. Whenever matches are discovered, this service notify subscriber, using asynchronous, push-based communication mechanisms (Echterhoff et al 2008). In this system SES is used for discovering emergency situations based on predefined criteria from input sensor observations. WNS is used as messaging service which providing asynchronous communication to registered user through sending an E-mail (Simonis et al 2003).

\section{CONCLUSION}

Sensor Web has provided infrastructure for collecting, sharing and processing data from spatially distributed and heterogeneous sensors. Accessing to real time, online and update information is a key issue in disaster management and early warning systems. The air quality monitoring system architecture which is described in this paper provides real time monitoring system for analyzing sensor observations and supports emergency response systems by providing real time, online and update air quality sensor observation and also discovering emergency situations by processing events and matching predefined criteria. Developed system, usage of standard SWE framework and by standardization of sensor observation into O\&M standard structure overcomes interoperability challenge and presents interoperable and flexible monitoring system. Air quality monitoring system by providing spatio temporal queries, has enable authorities to access their required observation in a standard based framework. Also by visualizing observation in time series chart provide capability of analyzing timely trend of air pollutants. In future work we want to provide spatial analysis and interpolation for estimating air quality information and air pollutant concentration in any point of interest which helps registered users in making more effective decision. Also uncertainty is a crucial issue which addressed in future study to improve air quality monitoring system event detection using reliable information extracted from sensor observations.

\section{REFERENCES}

Alesheikh, A., Oskouei, A., Atabi, F., Helali, H., 2005, providing interoperability for air quality in-situ sensors observations using GML technology.

Botts, M., Percivall, G., Reed, C., Davidson, J., 2006, “OGC Sensor Web Enablement: Overview and High Level Architecture", OGC White Paper, 06-046r2.

Bröring, A., Echterhoff, j., Jirka, S., Simonis, I., Everding, T., Stasch, C., Liang, S., Lemmens,R., 2011, New Generation Sensor Web Enablement, New Generation Sensor Web Enablement.Sensors, 11(3):2652-2699.

Delin, K.A., Jackson, S.P., 1999 Some, R.R. Sensor Webs, NASA Tech Briefs.

Echterhoff, J., Everding, T., 2008, OpenGIS Sensor Event Service Interface Specification, OGC 08-133.

Everding, T., Jirka, S., 2010, Combining Geospatial Data and Real-time Sensor Data for Calculating an Integrated Health Index

Henneböhl, K., Gerharz, L., Pebesma, E., 2009, An OGC web service architecture for near real-time interpolation of air quality over Europe.

Khedo, K., Perseedoss, R., Mungur, A., 2010, A Wireless Sensor Network Air pollution monitoring System, international jurnal of wireless and mobile networks, Vol2, No 2.

Markovic, N., Stanimirovic, A., Stoimenov, L., 2009, Sensor Web for River Water Pollution Monitoring and Alert System,

Na, A., Priest, M., 2007, Sensor Observation Service, OGC 06009 r6.

Resch, B., Britter, R., Outram, C., Chen, X., Ratti, C., 2012, Standardised Geo-Sensor Webs for Integrated Urban Air Quality Monitoring.

Schaeffer, B., 2009. OGC® OWS-6 Geoprocessing Workflow Architecture Engineering Report. (OGC Public Engineering Report No. 09-053r5) (p.78) OGC.

Schut, P., 2007 Web processing service v1.0.0. Implementation specification OGC 05-007r7, Open Geospatial Consortium Inc.

Simonis, I., 2011, Augmented Reality \& Sensor Web Enablement: A Position Paper $4^{\text {th }}$ AR Standards Community Meeting, Basel, Switzerland

Simonis, I., Wytzisk, A., 2003, Web Notification Service, OGC 03-008r2.

Stasch, C., Foerster, T., Autermann, C., Pebesma, E., 2011, Spatio-Temporal Aggregation of European Air Quality Observations in the Sensor Web.

7. 